\title{
EDITH STEIN
}

IN DER DEUTSCHEN

ERINNERUNGS-

UND GESCHICHTSKULTUR

In dem vorliegenden Beitrag geht es um die Frage, auf welche Weise Edith Stein in der Erinnerungskultur in Deutschland repräsentiert ist. Der deutsche Historiker Christoph Cornelißen definiert mit dem Begriff „Erinnerungskultur", , alle denkbaren Formen der bewussten Erinnerung an historische [...] Persönlichkeiten, seien sie ästhetischer, politischer oder kognitiver Natur"1. Dies alles im Zusammenhang mit Edith Stein aufzuzeigen, dazu reichen weder meine Facultas noch meine Kenntnisse aus. Vor allem, wenn man bedenkt, dass unter diesem umfassenden Anspruch auch die theologische und philosophische Rezeption berücksichtigt werden müsste. Mein Ansatz ist bescheidener. Er bezieht sich auf das visuelle Gedenken an Edith Stein in seiner realen Gegenständlichkeit. Das Gedenken zeigt sich in Denkmälern, Briefmarken oder in Patrozinien für Schulen. Ich möchte die Formen dieses Gedenkens in Deutschland skizzieren und an einigen Beispielen analysieren. Dabei wird es in erster Linie darum gehen zu zeigen, wer oder welche Gruppe die Person Edith Steins zu welchem Zweck perspektiviert und welches Bild dabei jeweils von dieser komplexen Persönlichkeit gezeichnet wird. Es wird dann zu diskutieren sein, für welche Diskurse Edith Stein in Anspruch genommen wird und ob diese Funktionalisie-

${ }^{1}$ Ch. Cornelißen, Was heißt Erinnerungskultur? Begriff - Methoden - Perspektiven, in: „Geschichte in Wissenschaft und Unterricht" 2003, No. 54, S. 548-563, hier S. 555. 
rung der historischen Person gerecht werden kann oder überhaupt gerecht werden will.

Lassen Sie mich zunächst etwas über meinen analytischen Rahmen sagen. Seit Ende der 1970er Jahre ist zunächst in der alten Bundesrepublik ein gesteigertes Interesse an Geschichte zu erkennen, das Klaus Bergmann 1993 mit einem schönen Satz beschrieb: „So viel Geschichte wie heute war nie!”2. Diese Feststellung gilt noch immer. Der anhaltende Boom von Geschichte schlägt sich in vielen beliebten Fernsehformaten nieder, aber man kann auch weitere Sachverhalte beobachten, seien es die allerorten stattfindenden Mittelaltermärkte, das Nachstellen von historischen Schlachten, historische Stadtführungen zu noch so entlegenen Orten, Computerspiele oder der touristische Geschichtsgebrauch ${ }^{3}$. Das alles führt dazu, die spezifische Leistung der Geschichtswissenschaft in den Hintergrund zu drängen oder wenigstens zu relativieren. Der bekannte Historiker und Geschichtsdidaktiker Jörn Rüsen konstatiert: „Das kritische methodische Denken im Umgang mit der Vergangenheit droht in der Bilderflut zu ertrinken, in der die Vergangenheit medial allenthalben präsent ist" ${ }^{\prime 4}$. Es erscheint also geboten, diesen ästhetischen und kreativen Umgang mit Geschichte und seine Produkte analytisch unter die Lupe zu nehmen. Denn nur auf diese Weise können die Strategien zur Herstellung von Geltungsansprüchen sichtbar gemacht werden.

Um den Geschichtsboom einem wissenschaftlichen Diskurs zugänglich zu machen, sind seit den 1970er Jahren zwei Konzepte entstanden, die das damit zusammenhängende Phänomen analytisch in den Griff zu bekommen trachten: die Erinnerungskultur und die Geschichtskultur ${ }^{5}$.

2 K. Bergmann, "So viel Geschichte wie heute war nie". Historische Bildung angesichts der Allgegenwart von Geschichte, in: Politische Sozialisation und Geschichte. Festschrift für Rudolf Schörken zum 65. Geburtstag, Angela Schwarz (Hrsg.), Hagen 1993, S. 209-228.

${ }_{3}^{3}$ Geschichtsdidaktik und Geschichtswissenschaft in Deutschland haben diesen Trend seit der Jahrtausendwende Jahren verstärkt aufgegriffen. Summarisch: Geschichte und Öffentlichkeit. Orte, Medien, Institutionen, S. Horn, M. Sauer (Hrsg.), Göttingen 2009; Die Anwesenheit von Vergangenheit in der Gegenwart, V. Oswalt, H.-J. Pandel (Hrsg.), Schwalbach/Ts. 2009; History goes Pop. Zur Repräsentation von Geschichte in populären Medien und Genres, B. Korte, S. Paletschek (Hrsg.), Bielefeld 2009; History sells! Angewandte Geschichte als Wissenschaft und Markt, W. Hartwig, Alexander Schug (Hrsg.), Stuttgart 2009.

${ }^{4}$ J. Rüsen, Historik. Theorie der Geschichtswissenschaft, Köln - Weimar - Wien 2013, S. 18.

${ }^{5}$ Vgl. die Kontroverse um die Begriffsinhalte: M. Demantowsky, Geschichtskultur und Erinnerungskultur. Zwei Konzeptionen des einen Gegenstandes. Historischer Hintergrund und exemplarischer Vergleich, in: „Geschichte, Politik und ihre Didaktik” 2005, No. 33, S. 11-20; B. Schönemann, Erinnerungskultur oder Geschichtskultur?, in: Kulturwissenschaften und Geschichtsdidaktik, E. Kotte (Hrsg.), München 2011, S. 53-72; vgl. auch H.-J. Pandel, Geschichtsdidaktik. Eine Theorie für die Praxis, Schwalbach/Ts. 2013, S. 161-177. 
Das Konzept der Erinnerungskultur meint nach Christoph Cornelißen, wie oben schon eingeführt, einen „formalen Oberbegriff für alle denkbaren Formen der bewussten Erinnerung an historische Ereignisse, Persönlichkeiten und Prozesse, seien Sie ästhetischer, politischer und kognitiver Natur". Der Autor fasst darunter "neben Formen des ahistorischen oder sogar antihistorischen kollektiven Gedächtnisses" alle möglichen Formen der Repräsentation von Geschichte, auch private Erinnerungen und den geschichtswissenschaftlichen Diskurs. Der Autor hebt dabei auf den funktionalen Gebrauch von Geschichte und Erinnerung für gegenwärtige Zwecke ab, etwa "für die Formierung einer historisch begründeten Identität" 6 . Insofern scheint dieser Zugang für die Frage nach dem visuellen Gedenken an Edith Stein geeignet zu sein. Der Bezug auf das ,kollektive Gedächtnis' macht dieses Konzept allerdings etwas problematisch. Denn die konkreten Träger dieses "Gedächtnisses", also die Subjekte der Erinnerung drohen in einem Kollektiv zu verschwimmen, dessen Konturen wenig erkennbar bleiben können. Gleichwohl hat der Gebrauch des Begriffs ,Erinnerungskultur' den entschiedenen Vorteil, dass er anschlussfähig an den memory- oder Gedächtnis-Diskurs erscheint, der nicht nur in allen Kulturwissenschaften derzeit äußerst attraktiv ist? .

Das Konzept der Geschichtskultur erscheint demgegenüber weniger attraktiv, ist aber analytisch wesentlich brauchbarer. Jörn Rüsen, einer der prominenten Vertreter dieses Konzeptes, sieht in der Geschichtskultur die äußere Seite einer "praktisch wirksamen Artikulation von Geschichtsbewusstsein im Leben einer Gesellschaft". ${ }^{8}$ Was heißt das? Die Geschichtskul-

6 Ch. Cornelißen, Erinnerungskulturen, Version: 2.0, in: "Docupedia-Zeitgeschichte", 22.10.2012, http://docupedia.de/zg/Erinnerungskulturen_Version_2.0_Christoph_Corneli. C3.9Fen?oldid=84892 [Abruf: 31.01.2014].

7 Der Begriff „,kollektives Gedächtnis” wird inflationär für alle möglichen Erinnerungsphänomene in der Gesellschaft gebraucht. Es geht dabei um die "Stiftung, Pflege und Reflexion des kulturellen Erbes" eines Kollektivs, zumeist einer Nation. Mit dem "kollektiven Gedächtnis" ist kein Sachgegenstand gemeint, sondern ein vorgestellter Ort, an dem das für ein Kollektiv sinnstiftende Geschichtsbild konstruiert wird, mit dem es sich selbst konstituiert. Als Ausgangspunkt der heutigen Überlegungen zum kollektiven Gedächtnis gelten die soziologischen Studien von Maurice Halbwachs zur mémoire collective und Aby Warburgs Arbeiten zum europäischen Bildgedächtnis in den 1920er Jahren. In den 1980er Jahren hat Pierre Nora das Gedächtnis-Thema mit seinen Erinnerungsorten (lieux de mémoire) in einem neuen und einflussreichen Konzept geformt. Aleida und Jan Assmann haben etwas später das „kollektive Gedächtnis” um ein „kommunikatives” und ein „kulturelles Gedächtnis” erweitert, um deutlich zu machen, dass Erinnerungen im Laufe der Zeit aus einem generationsgebundenen „kommunikativen” in ein „kulturelles Gedächtnis” übergehen. vgl. A. Erll, Kollektives Gedächtnis und Erinnerungskulturen. Eine Einführung, Stuttgart - Weimar 2. Aufl. 2011, S. 15-39.

8 Jörn Rüsen: Was ist Geschichtskultur? Überlegungen zu einer neuen Art, über Geschichte nachzudenken. In: Jörn Rüsen, Theo Grütter, Klaus Füßmann (Hrsg.), Historische Faszination. Geschichtskultur heute. Köln u.a. 1994, S. 3-26, hier S. 5. 
tur ist zunächst auf einen anderen Begriff, das Geschichtsbewusstsein, bezogen. Es sind gewissermaßen die zwei Seiten einer Medaille. Das Geschichtsbewusstsein wird als ein mentaler Ort verstanden, an dem sich ein Individuum mit den Repräsentationen der Geschichtskultur auseinandersetzt und sich dazu in Beziehung setzt ${ }^{9}$. In diesem Prozess wird, so Rüsen, ein spezifischer historischer Sinn gestiftet. Das Individuum gibt einem historischen Ereignis, einer Persönlichkeit einem Prozess historischen Sinn. Dabei entstehen Produkte, zum Beispiel Denkmäler, Briefmarken, Filme oder andere historische Repräsentationen, die man zusammenfassend als Geschichtskultur bezeichnet. Diese Sinnstiftung kann nach Rüsen in eine kognitive, eine politische und eine ästhetische Dimension unterschieden werden. Diese treten nicht gegen- oder nebeneinander auf, sondern sind zumeist in spezifischen Mischungsverhältnissen vorhanden. Dieses hier in groben Zügen beschriebene Konzept bietet mit dem Schlüsselbegriff der Sinnstiftung die geeignetere Zugangsweise, weil dieser Sinn auf verschiedene Kriterien untersucht werden kann, die seinen Geltungsanspruch begründen. Im kognitiven Bereich nach empirischen Wahrheitskriterien, im politischen nach Machtkriterien und im ästhetischen nach Schönheitskriterien.

Legt man ein solches Verständnis von Geschichtskultur zugrunde, können der Person Edith Steins Aspekte abgewonnen werden, die in der gängigen Rezeption bislang kaum berücksichtigt worden sind. Bezeichnend ist ein Blick in das Buch von Francesco Alfieri von 2012 mit dem Titel Die Rezeption Edith Steins ${ }^{10}$. Die von mir hier angedeuteten Rezeptionsgedanken sind Alfieri völlig fremd. Die vorgelegte Bibliographie beschäftigt sich ausschließlich mit der geistesgeschichtlichen Rezeption der Schriften Edith Steins mit dem Ziel, die Bedingungen aufzuzeigen, um Steins Werk zu verstehen: „the understanding of the person within a research community"11. An dieser Bestimmung wird schon deutlich, dass hier das, was eine Person ausmacht und was ihre Rezeptionsgeschichte bestimmt, ziemlich eng gefasst ist.

Es ist überhaupt auffällig, dass die im engeren Sinn historische Erforschung ihres Lebens nicht zu den Schwerpunkten der Edith-Stein-Forschung zu gehören scheint. So finden sich nur wenige Titel, in denen das Leben der Protagonistin im Zentrum steht ${ }^{12}$. Bisweilen werden dort auch Aspekte zur

${ }_{9}^{9}$ Der Begriff Geschichtsbewusstsein gilt in Deutschland als „zentrale Kategorie” der Geschichtsdidaktik.

${ }^{10}$ F. Alfieri, Die Rezeption Edith Steins. Internationale Edith-Stein-Bibliographie 1942-2012. Festgabe für M. Amata Neyer OCD.Würzburg 2012.

${ }^{11}$ R. Miron, Rezension, in: „Iyyun. The Jerusalem Philosophical Quarterly”, No. 62 (January 2013), S. 98-104.

12 W. Herbstrith, Edith Stein. Jüdin und Christin. Ein Porträt, München 4. Aufl. 2004; Ch. Feldmann, Edith Stein: Biographie, Reinbek b. Hamburg 2. Aufl. 2004; A.U. Müller, M.A. 
gesellschaftlichen Wirkungsgeschichte skizziert. Sie werden aber kaum systematisch erschlossen. Im Vordergrund steht ihre ideengeschichtliche Wirkungsgeschichte, hinter der die historische Person bisweilen zu verschwinden droht. So eröffnet Gisela Lange in einem Grußwort zur Digitalisierung des Edith-Stein-Archivs ihre Ausführungen zum Leben Edith Steins mit einem Rilke-Zitat: „Schicksal ist nichts als das Dichte der Kindheit”. Sie fährt fort: „Dieses Rilke Wort trifft auf Edith Stein zu. Sie wurde am 12. Oktober 1891, dem großen Versöhnungsfest der Juden, in Breslau geboren. An diesem Tag durfte der Hohepriester durch ein Opfer die Sünden des Volkes tilgen. In der christlichen Liturgie ist dieser Tag vergleichbar mit Karfreitag und Ostern. Und gerade diese Symbolik des Opfertieres, des holocaustum, bietet den Schlüssel für ihre Identität"13. Ich will nicht sagen, dass diese Deutung gänzlich unzutreffend ist. Man muss jedoch betonen, dass solche ex post in die Kindheit hineingelegte Lebensprogramme die Kontingenzen tilgen, die ein Leben normalerweise ausmachen. Selbstverständlich ist das Leben Edith Steins so verlaufen, wie es verlaufen ist. Aber wenn das Schicksal ins Spiel kommt, geht der einfache Gedanke verloren, dass sie sich an verschiedenen Stellen ihres Lebens auch hätte anders entscheiden können.

Sie steht uns, so Gisela Lange, „als Jüdin, als christliche Philosophin und Karmeliterin, Mystikerin sowie Märtyrin" vor Augen. Das ist sicher richtig. Aber wie steht sie uns als Frau, als Tochter, als Arbeitskollegin, als Freundin vor Augen? Wäre das zu wissen nicht ebenfalls für eine Bewertung ihres Lebens wichtig? Ihre Seligsprechung im Jahr 1987 und ihre Heiligsprechung im Jahr 1998 haben Antworten auf solche Fragen nicht eben leichter gemacht.

Insofern wundert es kaum, dass Edith Steins Präsenz in der deutschen Geschichtskultur eher von ihrer überwältigenden Lebensdramatik, ihrem scheinbar unbeirrbaren Festhalten an Lebenszielen, ihrer Überzeugung von der Leistung von Bildung und ihrem Glauben an die Kraft der Verknüpfung von philosophischen und theologischen Einsichten geleitet ist. Damit wird sie im Grunde enthistorisiert, ja entpersonalisiert und den Bedürfnissen und Funktion der Produzenten der geschichtskulturellen Produkte angepasst. Ohne einen Anspruch auf Vollständigkeit zu erheben, manifestiert sich ein derartiges Gedenken an Edith Stein in Denkmälern ${ }^{14}$, Briefmarken ${ }^{15}$, Patro-

Neyer, Edith Stein, Das Leben einer ungewöhnlichen Frau, Düsseldorf 2002; E. Endres, Edith Stein. Christliche Philosophin und jüdische Märtyrerin, München 1987.

${ }^{13}$ G. Lange, Grußwort zur Digitalisierung des Edith-Stein-Archives. Online im Internet:

URL: www.edith-stein-archiv.de/grusswort_zu_beginn_der_digitalisierung.pdf [Abruf: 31.01.2014].

${ }^{14}$ Figurative Edith-Stein-Denkmäler gibt es in Köln (1999, Künstler: Bert Gerresheim), in Berlin (2009, Künstler: Bert Gerrisheim) und in Landau (2008, Künstler: Peter Brauchle). Eine 
zinien für Schulen ${ }^{16}$, in Gedenktafeln an ehemaligen Wohnstätten ${ }^{17}$, in Spielfilmen ${ }^{18}$, in unzähligen Edith-Stein-Straßen, -plätzen, -anlagen in unterschiedlichen Städten in ganz Deutschland und in zahlreichen Edith-SteinKliniken und Kirchen sowie im seit 1995 verliehenen Edith-Stein-Preises ${ }^{19}$.

Nun zu einigen Beispielen. Das 1999 vor dem Priesterseminar in Köln aufgestellte Denkmal (Abb.1) stammt von dem Künstler Bert Gerresheim. Gerresheim, 1935 in Düsseldorf geboren und hauptberuflich Lehrer, schuf zahlreiche Plastiken. "Als gläubiger Christ und Katholik setzt sich Gerresheim vor allem mit christlichen Themen auseinander. Seine Passionsszenen greifen die Leiden von Krieg und Verfolgung auf und schaffen so eine unmittelbare Verbindung der Leiden Christi mit der modernen Welt." 20 In dem Denkmal stellt er die drei zentralen Stationen des Lebens von Edith Stein, wie er sie versteht, in den Mittelpunkt seiner Plastik: als Jüdin, Philosophin und Karmelitin. Der Künstler zeigt Stein sitzend als junges Mädchen mit einem Davidstern, stehend als Philosophin und als Karmelitin, den Gekreuzigten tragend ${ }^{21}$. Interessant ist die Deutung der mittleren Figur, deren Kopf gespalten ist. Sie hält einige ihrer Schriften unter dem Arm. Erkennbar sind zum Beispiel „Zum Problem der Einfühlung”, ihre philosophische Dissertation, und eines ihrer Hauptwerke: die "Kreuzeswissenschaft”. Die Symbolik des gespaltenen Kopfes ist nicht leicht zu deuten. Ich finde es jedoch abwegig, wie ich es zum Teil gelesen habe, von einer "gespaltenen

Büste (Künstler: Johann Brunner) wurde 2009 in der Walhalla aufgestellt. Seit 2006 steht eine Marmor-Statue in einer Seitenkapelle in der Peterskirche in Rom (Künstler: Paul Nagel)

${ }^{15}$ Anlässlich ihrer Ermordung in Auschwitz gab die Bundespost 1983 eine Briefmarke heraus (Michel-Katalog-Nr. 1162). Eine zweite Marke erschien im Zusammenhang mit ihrer Seligsprechung 1987. Auf dieser Marke ist sie zusammen mit dem Jesuiten Rupert Mayer abgebildet, der im gleichen Jahr seliggesprochen wurde (Michel-Katalog-Nr. 1352).

${ }^{16}$ Es war nicht möglich, auch nur eine annähernde Zahl der Edith-Stein-Schulen in Deutschland zu ermitteln. Stichprobenartig zeigt sich aber eine Konzentration in den überwiegend katholischen Gebieten.

${ }_{17}$ Gedenktafeln finden sich an der Stelle von Steins ehemaligen Karmel in Köln, Dürer Straße (http://commons.wikimedia.org/wiki/File:Koeln-Edith-Stein-Gedenktafel.jpg), in Göttingen, Lange-Geismar-Straße (http://www.edith-stein-kreis.de/edith-stein-in-goe.htm) und außerhalb Deutschlands in Wrocław (http://commons.wikimedia.org/wiki/File:Dom EdytyStein.jpg).

${ }_{18}$ Die Jüdin Edith Stein (Orig.: Siódmy pokój, Spielfilm I/H/PL/F/D 1995, 97 min). Online im Internet: URL: http://www.imdb.com/title/tt0114461/ [Abruf: 31.01.2014].

${ }^{19}$ Edith-Stein-Preis. Informationen des Göttinger Edith-Stein-Kreises. Online im Internet: URL: http:/ / www.edith-stein-kreis.de/edith-stein-preis.htm [Abruf: 31.01.2014].

${ }_{20}$ Bert Gerresheim. In: Wikipedia. Online im Internet: URL: http://de.wikipedia.org/ wiki/Bert_Gerresheim [Abruf: 31.01.2014].

${ }^{21}$ Informationen zum Denkmal auf einer Seite des Georg-Büchner-Gymnasiums. Online im Internet: URL: http://www.gbg-koeln.de/denkmal/edith/ablass.htm [Abruf: 31.01.2014]. 
Persönlichkeit" zu sprechen. Plausibler als Erklärung scheint mir das in der „Kreuzeswissenschaft” angesprochene Problem zu sein, wissenschaftliche und Glaubenswahrheiten miteinander zu kombinieren. Richard Niedermeyer brachte das 2003 in einer Rezension auf folgende Formel: „Lässt sich der Inbegriff menschlichen Leidens und zugleich ein Kernmoment des christlichen Mysteriums überhaupt wissenschaftlich darstellen?"22.

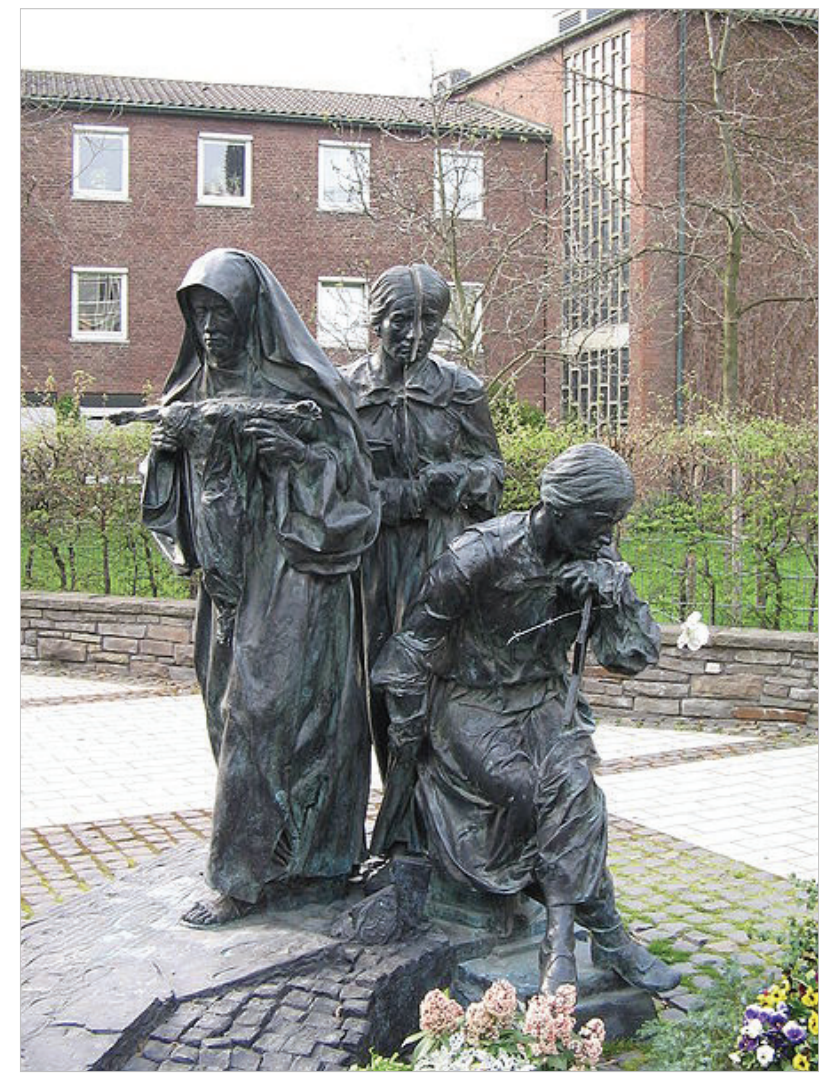

Abb. 1: Edith-Stein-Denkmal im Innenhof des Priesterseminars in Köln (Quelle: http://commons.wikimedia.org/wiki/File:Holocaust_denkmal_detail_k\%C3\%B6ln.jpg)

Abseits dieses Deutungsproblems geht es mir noch um etwas Anderes. Es gibt im Hinblick auf Edith Stein nicht nur eine Narration, sondern mehrere. Die Sichtweise des christlichen Künstlers und des Kölner Erzbistums, das

22 R. Niedermeyer, Rezension: Edith Stein: Kreuzeswissenschaft. Studien über Johannes vom Kreuz (1942), Freiburg 2003, in: Buchkritik.at. Online im Internet: URL: http://www. buchkritik.at/kritik.asp?IDX=1981. 
den Auftrag zu der Herstellung der Plastik gegeben hatte, traf im Rahmen der Realisation des Projekts auf eine andere. Viele Bürger der Stadt Köln mochten in dem Denkmal keine angemessene Darstellung Edith Steins erkennen. Sie befürchteten, dass die Ermordung der Jüdin Edith Stein im Vernichtungslager zu einem christlichen Opfer umgedeutet werden könne. Das Bistum erhielt deshalb von der Stadt keine Genehmigung, das Denkmal im öffentlichen Raum aufzustellen. Stattdessen wurde es auf dem Grundbesitz des Bistums vor dem Priesterseminar errichtet ${ }^{23}$.

An solchen Auseinandersetzungen wird deutlich, dass der Bereich der Geschichtskultur häufig nicht der Ort von großen Differenzierungen ist ${ }^{24}$. Während sich der Künstler vielleicht noch bemüht hat, die verschiedenen Facetten der Person herauszuarbeiten, ließ Kardinal Meisner in seiner Rede bei der Enthüllung des Denkmals gar keinen Zweifel daran, dass er Edith Stein für eine christliche Märtyrin halte:

Köln ist nicht nur die Stadt des Karnevals, sondern auch die Stadt der Märtyrer. Das schließt einander nicht aus, sondern ein. Der Apostel bezeugt uns ausdrücklich: Durch das Holz des Kreuzes kam Freude in unsere Welt. Wir stehen hier innerhalb der Stadt in der geographischen Mitte zwischen den altehrwürdigen Märtyrerkirchen St. Gereon und St. Ursula. In der Ersteren verehren wir die Reliquien der thebäischen Legionäre, in der anderen die Reliquien der hl. Ursula. Wir weihen heute eine neue Märtyrerstätte ein, das Denkmal für die hl. Edith Stein oder die hl. Karmelitin, Schwester Theresia Benedicta a Cruce. Sie ist keine Märtyrerin der Vergangenheit, sondern der Gegenwart. Moderner Vernichtungswille mit seinen technischen Möglichkeiten arbeitete so gründlich, dass er uns keine Reliquien mehr zurücklässt. Für die Märtyrer unserer jüngsten Geschichte gibt es daher keine Reliquienschreine. Wer will uns dann verübeln, dass wir ihnen und uns eine Gedächtnisstätte, oder wie man profan sagt - ein Denkmal errichten. Wir brauchen solche sichtbaren Zeichen der Anwesenheit unserer Märtyrer auf den Wegen der Gegenwart. Sie werden uns zur Ermutigung, wenn wir sie sehen, an ihnen vorübergehen oder bei ihnen still verharren. Sie bringen uns in Berührung mit ihrem sieghaften Glauben ${ }^{25}$.

Er ordnete den Standort des Denkmals geographisch zwischen den Märtyrerkirchen St. Gereon und St. Ursula ein. Von der Sache stellte er die Hei-

${ }^{23}$ Informationen zum Denkmal (wie Anm. 21).

${ }^{24}$ Der Problematik, ob Edith Stein eine christliche oder jüdische Märtyrerin sei, widmen sich aus christlicher Perspektive Heinrich Mussinghoff: Laudatio anläßlich der Verleihung der Edith-Stein-Medaille an Landesrabbiner Dr. Henry G. Brandt am 30. Oktober 2011 in Göttingen, in: „Edith Stein Jahrbuch” 2012, No. 18, S. 26-41; aus jüdischer Perspektive: Henry G. Brandt: Rede von Landesrabbiner Dr. h.c. Henry G. Brandt, dem Edith-Stein-Preisträger 2011, zur Preisverleihung am 30. Oktober 2011, in: ebenda, S. 42-48.

${ }^{25}$ Informationen zum Denkmal (wie Anm. 21). 
lige Edith Stein neben die Heilige Ursula. Das Denkmal bezeichnete er als Ersatz für die Reliquien, die frühere Zeiten im Gegensatz zu dem „modernen Vernichtungswillen" noch hinterlassen hätten. Diese Sichtweise mag aus der Perspektive eines katholischen Kardinals nachvollziehbar sein, eine angemessene Beschreibung der Person Edith Steins ist sie sicher nicht.

Ganz anders die Ansprache der damaligen Ministerin Anette Schavan 2009 bei der Enthüllung eines zweiten Denkmals für Edith Stein in Berlin, das abermals vom Künstler Gerresheim geschaffen wurde:

Bert Gerresheim, der die Skulptur von Edith Stein geschaffen hat, regt zur Auseinandersetzung mit dieser Frau an, mit ihren Texten, ihrer Philosophie, mit ihrem Glauben und ihrem Lebensweg. Diese Skulptur ist ein Denkmal, das auch Mahnmal ist. Edith Stein ist ein Opfer des Holocaust. Eingebrannt in unsere Erinnerung sind ihre Worte zu ihrer Schwester bei der Verhaftung: „Komm, wir gehen für unser Volk.” Das Denkmal der Heiligen Edith Stein erinnert uns an den Versuch der Nationalsozialisten, ein ganzes Volk auszulöschen. Sie sind weit damit gekommen, den Deutschen das Gewissen auszutreiben. Die Shoa war der unvergleichliche Verstoß gegen die Zertrümmerung jedweder ethischer Prinzipien, die seit jeher weltweit alle Kulturen und Religionen verbinden. Der Versuch, alle Moral, jedes Gefühl für Gut und Böse abzuschaffen ${ }^{26}$.

Der Künstler rege zur Auseinandersetzung mit Edith Stein an. Schavan sieht in der Skulptur sowohl ein Denkmal als auch ein Mahnmal. Edith Stein sei ein Opfer des Holocaust. Den Satz, den Edith bei der Verhaftung zu ihrer Schwester gesagt haben soll, hebt die Ministerin stark hervor: „Komm, wir gehen für unser Volk." Hier wird nun eine ganz andere Perspektive als die des katholischen Kardinals deutlich. Es ist die Perspektive der Staatsraison der Bundesrepublik Deutschland. Das Gedenken an Edith Stein wird als mahnendes Beispiel für die Folgen der Ausrottungspolitik des nationalsozialistischen Staates gedeutet. Diese Erinnerung, so Schavan, sei ein Wegweiser aus der Vergangenheit in die Zukunft. Die Ermordung der Jüdin Edith Stein soll daran erinnern, dass Demokratie und Menschenrechte jeden Tag aus Neue verteidigt werden müssen. Interessant ist in diesem Zusammenhang, dass das neue Denkmal von 2009 nur noch einen Aspekt des Kölner Denkmals von 1999 aufgreift.

${ }^{26}$ A. Schavan, „Wer die Wahrheit sucht” - Erinnerungskultur beleben. Grußwort der Bundesministerin für Bildung und Forschung, Prof. Dr. Annette Schavan, MdB, anläßlich der Enthüllung des Denkmals zu Ehren von Edith Stein am 25. März 2009 in Berlin, in: „Tabula Rasa. Zeitschrift für Gesellschaft und Kultur" No. 39 (5/2009). Online im Internet: URL: http://www.tabularasajena.de/artikel/artikel_904/ [Abruf: 31.01.2014] 


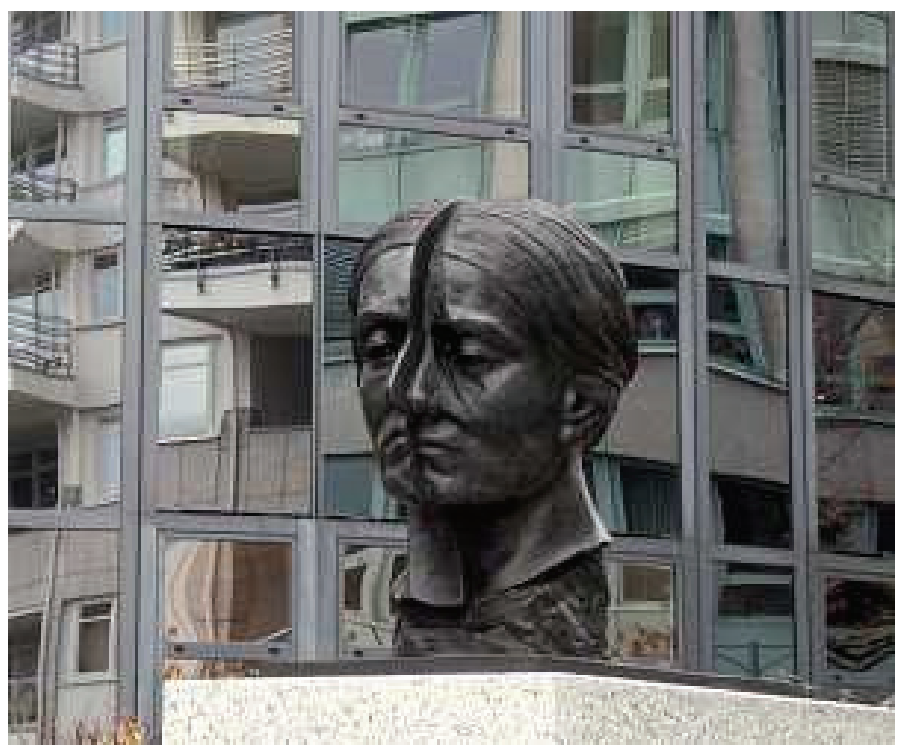

Abb. 2: Büste der Edith Stein in Berlin, Straße der Erinnerung in Moabit (Quelle: @ -wn-. In: Sehenswürdigkeiten in Berlin. Online im Internet: URL: http://www.in-berlin-brandenburg.com/Sehenswuerdigkeiten/Gedenkstaetten/ Strasse-der-Erinnerung.html)

Ähnliche Vereinnahmungen finden wir in anderen Repräsentationen der Geschichtskultur. 2010 wurde Edith Stein auf Beschluss der bayerischen Staatsregierung in die Walhalla aufgenommen ${ }^{27}$. Seit 1842 werden dort Büsten bedeutender Persönlichkeiten "teutscher Zunge” nach ihrem Tod präsentiert. Ursprünglich ein rein nationales Projekt bemüht sich die bayerische Staatsregierung, diese Vorbehalte durch eine pluralistische Ausrichtung der Geehrten zu zerstreuen. Die Büste selbst ist zwar wieder vollständig in den katholischen Kontext eingeordnet, aber die Süddeutsche Zeitung berichtete am 7. Mai 2010 unter der Überschrift „Edith Stein in der Walhalla: Die heilige Nazi-Gegnerin” gut pluralistisch: „Ein neuer Marmorkopf in der Walhalla: Seit heute steht die Büste der in Auschwitz ermordeten Nonne Edith Stein in der Gedenkstätte. Sie war die erste jüdischstämmige Heilige Europas"28. Dieses Narrativ ebnet schließlich alles ein: es bleiben nur noch die Nazis als das Böse und die Identifikationsfiguren, die sich gegen eben dieses

${ }^{27}$ H. Hanske, J. Traeger, Walhalla. Ruhmestempel an der Donau, Regensburg 1998.

${ }^{28}$ Die heilige Nazi-Gegnerin. In: Süddeutsche.de. Online im Internet: URL:

http://www.sueddeutsche.de/bayern/edith-stein-in-der-walhalla-die-heilige-nazigegnerin-1.106828

[Abruf: 31.01.2014]. 
Böse aufgelehnt haben. Ganz gleich, ob es Juden, Katholiken oder andere aufrechte Europäer waren. Diese erscheinen als freiheitliche und pluralistische Demokraten, die eine tief empfundene Menschlichkeit, wurzelnd in den Menschenrechten der französischen Revolution gegen die Unmenschlichkeit der Nazis in Stellung gebracht haben. Über alle Differenzen hinweg wird mit diesen Nazi-Gegnern ein Bündnis geschlossen, so dass am Ende alle möglichen Gruppen als Opfer des nationalsozialistischen Terrors gedeutet werden können.

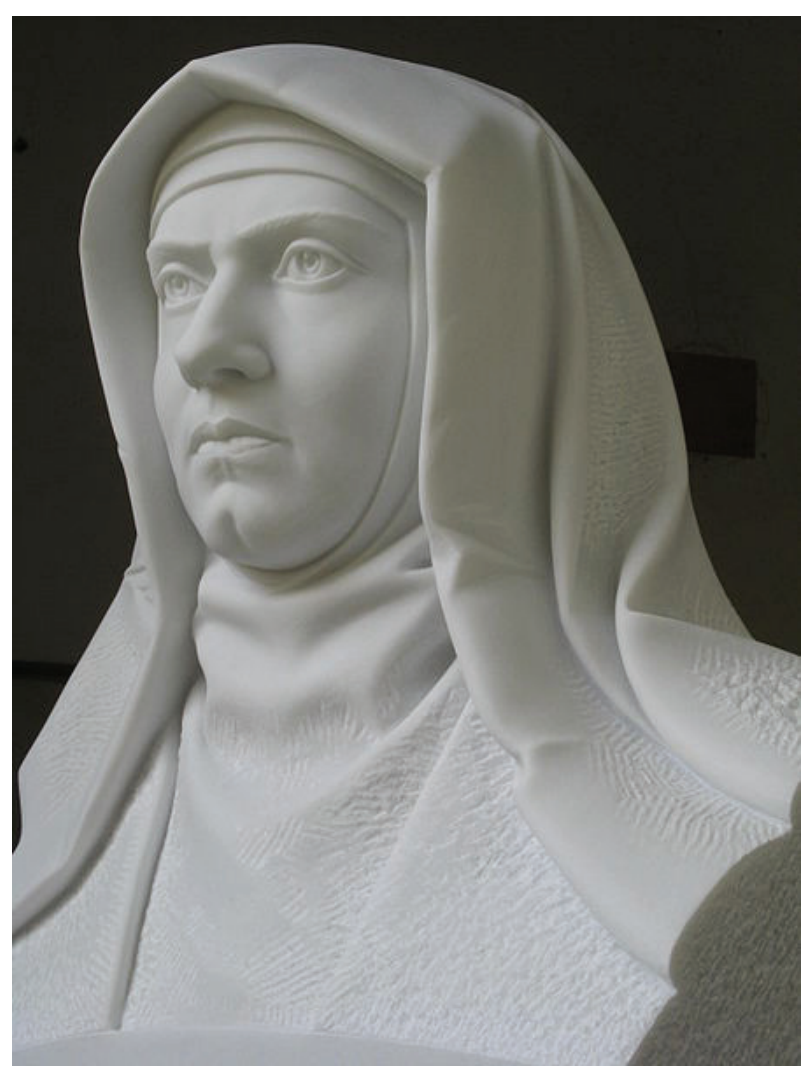

Abb. 3: Büste der Edith Stein in der Walhalla, Regensburg. Material: Laaser Marmor, Bildhauer: Johann Brunner, Surberg. Erstellungsjahr: 2008

(Quelle: http://commons.wikimedia.org/wiki/File:Edith_Stein_Ausdruck.JPG)

Eine ganz ähnliche Tendenz findet sich bei den Patrozinien der zahlreichen Edith-Stein-Schulen, die sich bei der Namensgebung ganz bewusst wieder nur auf bestimmte Aspekte beziehen, die sie bei ihrer Bildungsarbeit 
in den Mittelpunkt stellen wollen. Die in der Tat häufig katholischen Schulen sehen Edith Stein einerseits als glaubhafte Vertreterin eines „christlichen Menschenbildes" 29 oder als jemanden, der "aus tiefster Überzeugung zum katholischen Glauben übertrat" 30 . Einige Schulen sprechen von der Toleranz, die Edith Stein vermittele, und davon, dass Edith Stein „über den Tod hinaus für Versöhnung steht, für Versöhnung unter den Menschen und Versöhnung unter den Religionen"31. Auch hinter diesen Aussagen kann man eine Vereinnahmung für gegenwärtige Zwecke erkennen. Man greift eben nur bestimmte Aspekte heraus und ordnet die Protagonistin auf ein bestimmtes Ziel hin, das gegenwärtigen Wertvorstellungen entspricht. Dazu passt auch, dass einige Schulen Edith Stein als „Pionierin eines modernen Frauenbildes” erkennen. Sie helfe angeblich „bei der Suche danach, wie Aufgaben als Ehefrau, Mutter und Berufstätige miteinander verbunden werden können" 32 . Mir erschließt sich nicht wirklich, welches Leitbild Edith Stein dabei abgeben soll.

In der geschichtskulturellen Repräsentanz kann man erkennen, dass sich im Laufe der Zeit das, was man mit Edith Stein in Verbindung bringt, immer mehr von ihrer Person, ihrem Leben abkoppelt. Heute scheint es fast so, dass ihre Person zu einem Symbol geworden ist, das ganz verschiedene Gruppen mit den ihnen gemäßen Wertvorstellungen verknüpfen können. Ich habe große Zweifel, dass man Edith Stein damit gerecht wird.

\section{The Representation of Edith Stein in Contemporary} German Historical Culture

\section{Summary}

The article deals with the representation of Edith Stein in contemporary German historical culture. Being complex, Edith Stein's biography is demanding to de-

${ }^{29}$ Edith-Stein-Schule Erfurt: „Das christliche Menschenbild ist Orientierung und Ziel”. Online im Internet: URL: http://www.ess-erfurt.de/index.php?action=show_page\&id= 115\&mnuID=139 [Abruf: 31.01.2014].

${ }^{30}$ Edith-Stein-Berufskolleg Paderborn: „Als Jüdin, die aus tiefster Überzeugung zum katholischen Glauben übertrat." Online im Internet:

URL: http://www.edith-stein-berufskolleg.de/esb/index.php?ber_id=4025\&inh_id= 15657 [Abruf: 31.01.2014].

${ }^{31}$ Edith-Stein-Schule Freiburg im Breisgau: „Verehrte Lehrerschaft der Edith-Stein-Schule, Sie haben aus gutem Grund Ihrer Schule den Namen dieser Frau gegeben, an der unser Jahrhundert zutiefst schuldig wurde, die aber über den Tod hinaus für Versöhnung steht, für Versöhnung unter den Menschen und Versöhnung unter den Religionen." Online im Internet: URL: http://www.hls-freiburg.de/\#\{2\} [Abruf: 31.01.2014]

32 Edith-Stein-Berufskolleg Paderborn (wie Anm. 30). 
scribe. Finding a narrative, which would embrace her whole live in a comprehensible way, is therefore a formidable task. The analysis of her representation in German historical culture has revealed that different groups in the German society use certain images of Stein for their own purpose. They choose a convenient part of her life that best supports the purposes of their specific project, be it a school, clinic, church, et cetera. Thus Edith Stein becomes a quarry in which everybody can find what they need to find. 